\title{
Commentaire
}

\section{Biodiversité amazonienne : cet étrange objet du savoir}

\author{
Florence Pinton \\ Sociologue, AgroParisTech, 75005 Paris, France
}

L'homme est-il créateur de biodiversité ? Sujet ambitieux et risqué auquel s'attaque Florent Kohler ${ }^{1}$. Viennent immédiatement à l'esprit les questions d'échelle de temps et d'espace où situer le raisonnement, les référentiels à prendre en considération, voire ce qu'on entend par biodiversité et comment on la compte. Pour traiter de cela, F. Kohler propose de discuter la corrélation établie entre biodiversité et diversité culturelle. Il adosse sa dissertation au contexte brésilien en se référant aux populations amazoniennes, dont on sait qu'elles ont bénéficié d'un mouvement politique particulier, le mouvement socioenvironnemental, pour obtenir des droits fonciers sur des portions d'espaces forestiers à protéger. Ce mouvement défend l'idée que les populations autochtones et traditionnelles disposent d'organisations et de connaissances favorables à l'aménagement et à la gestion des milieux naturels. En d'autres termes, c'est plus au nom d'un principe de proximité avec la nature que d'un principe de réalité que s'est opéré le rapprochement que F. Kohler entend dénoncer. Mais c'est d'abord à sa discipline qu'il s'en prend, en l'occurrence l'anthropologie, qui, écrit-il, "pose comme allant de soi que les cosmologies "natives" sont respectueuses de l'environnement et génératrices de biodiversité ». Ce sont, d'une certaine façon, les fondements de la construction des aires protégées au Brésil, sous contrôle de populations résidentes, qu'il remet en cause.

Le texte de F. Kohler est stimulant quand il dénonce des lieux communs (les fausses représentations que l'on peut avoir de l'attitude des populations traditionnelles vis-à-vis de leur environnement) et surtout parce qu'il propose, dans sa deuxième partie, des outils d'analyse

Auteur correspondant : florence.pinton@agroparistech.fr

${ }^{1}$ Voir dans ce numéro l'article de Florent Kohler, « Diversité culturelle et diversité biologique : une approche critique fondée sur l'exemple brésilien ». pour décrypter les différents registres d'action des communautés enquêtées et ainsi évaluer leur disposition à gérer durablement la biodiversité. L'expérience montre en effet que le raccourci souvent fait entre mode traditionnel de gestion des ressources et conservation peut être source de dérives, car il repose sur une vision idéalisée du comportement des populations impliquées et de leur organisation communautaire. Les actions de développement se limitent alors à un transfert de pouvoir à des groupes dont les pratiques sociales ont été considérées comme immuables et instituées collectivement. Mais, au-delà de leur contenu, l'articulation des deux parties laisse perplexe dans la mesure où la posture de recherche adoptée par l'auteur pour chacune d'entre elles ne s'inscrit pas, me semble-t-il, dans la même ontologie. Autrement dit, c'est dans le cadre d'une pensée naturaliste que F. Kohler s'insurge contre la lecture anthropocentrée de la biodiversité et ses dérives, pour ensuite rompre avec elle et lui préférer une pensée environnementale tournée vers l'apprentissage et la quête de nouveaux paradigmes. C'est ce décalage qui je me propose de commenter.

Le cadre analytique de la première partie ( Nouveau paradigme ou pétition de principe? ») me semble parfois contestable ou maladroitement posé. F. Kohler reprend à son compte la pensée duale portée par la modernité, celle de l'objet et du sujet, qui donne aussi un sens à l'histoire, celui de la construction de sociétés humaines qui s'arrachent à la nature. À trop vouloir considérer la preuve scientifique comme unique source de vérité, il fait siennes nombre d'oppositions binaires pourtant affaiblies aujour$\mathrm{d}$ 'hui entre faits indiscutables et valeurs, entre nature et culture, entre science et politique, alors que le cas du Brésil, sur lequel il s'appuie, est l'exemple même d'une hybridation reconnue de ces différentes sphères. À l'interface d'un objet scientifique et d'un outil de gestion de l'espace, les aires protégées sont aussi des instruments 
politiques. Leur histoire s'inscrit dans des dynamiques de contrôle, de gestion, voire d'intégration des sociétés, depuis les politiques coloniales jusqu'aux actions participatives. Les enjeux politiques sont énormes et l'anthropologue peut difficilement éviter de les traiter. F. Kohler l'admet implicitement dans sa deuxième partie ( Sur la méthode anthropologique ») quand il soulève «la question de l'avenir des aires protégées [.. . ] face à une croissance humaine qui sera toujours, en fin de compte, tenue pour prioritaire».

Arrêtons-nous maintenant sur cette première partie, qui illustre le parti pris de l'auteur. L'approche de la biodiversité défendue est à la fois séduisante et contestable. Séduisante car F. Kohler revendique une vision biocentrée de la biodiversité qui cherche à se démarquer de la figure du sauvage naturalisé puis naturaliste dénoncée par Philippe Descola : "Alors que le naturalisme $\mathrm{du} \mathrm{XVIII}^{\mathrm{e}}$ se refusait à reconnaître une existence sociale aux Indiens, l'écologie culturelle contemporaine les crédite d'une admirable clairvoyance dans la sélection qu'ils opèrent des institutions les plus adaptées à leur habi$\operatorname{tat}^{2}$. » Mais il procède ensuite à une série d'affirmations que même les naturalistes auraient des difficultés à valider sans sourciller. Que sait-on de l'impact de la disparition d'un élément de la biodiversité sur l'équilibre d'un système, sinon que les exigences de régulation de systèmes complexes restent soumises à controverses tandis que les connaissances scientifiques ne sont pas unanimement partagées. Et comment confronter ces dynamiques au processus d'érosion de la diversité culturelle? On se trouve au-delà des limites de la connaissance scientifique et s'y référer crée le trouble plus que le doute. Ce qui semble acquis, c'est que l'assimilation entre les deux types de diversité, quand elle se fait, est plus de l'ordre de la rhétorique que de l'argumentation scientifique. La politisation de la biodiversité ne permet pas d'échapper à ce type de discours. Elle coïncide avec la diffusion d'une nouvelle réflexivité de la pensée occidentale sur l'histoire naturelle, les sciences et la technologie et la constitution de nouveaux objets de gouvernement - biodiversité, déforestation, changement climatique - dans lesquels les populations locales et leur savoir sur la nature sont mis à contribution. Ne doit-on pas concevoir la construction $\mathrm{du}$ lien entre diversités biologique et culturelle comme une tentative de rupture avec l'ontologie des modernes?

F. Kohler s'emploie à pointer les dérives induites par la construction d'une « vision culturalisée de la nature », véhiculée à l'origine, selon lui, par l'écologie culturelle ; ses travaux auraient contribué - l'ouvrage de W. Balée sur l'Amazonie est cité - à déléguer la gestion de la forêt amazonienne à des populations peu soucieuses de

\footnotetext{
${ }^{2}$ Descola, P., 1985. De l'Indien naturalisé à l'Indien naturaliste : sociétés amazoniennes sous le regard de l'Occident, in Cadoret, A. (Ed.), Protection de la nature : histoire et idéologie. De la nature à l'environnement, Paris, L'Harmattan, p. 233.
}

sa conservation. Il aurait été intéressant de reprendre les différentes filiations issues de cette école de pensée pour en saisir la complexité et la portée réelle, au moins en ce qui concerne l'Amazonie. On sait que l'écologie culturelle s'est constituée en réaction à l'évolutionnisme hérité de l'histoire coloniale pour mettre un terme à l'approche strictement naturaliste des Amérindiens. La prise en compte des contraintes écologiques comme facteur influençant les sociétés humaines a connu son véritable essor avec les travaux de Julian $H$. Stewart ${ }^{3}$, pour qui l'écologie du bassin amazonien introduisait une limite indépassable au niveau de développement que pouvaient atteindre les autochtones. Sous son influence, de multiples tentatives ont été faites pour réduire la compréhension des institutions amérindiennes (guerre, nomadisme, tabous alimentaires) à des fonctions adaptatives aux facteurs limitants de l'écosystème, tels que la rareté des protéines animales, la dispersion des ressources, l'envahissement des abattis par les adventices ou encore le renouvellement de la fertilité du sol. À la même époque, Harold Conklin, jeune anthropologue américain, proposait de partir des catégories sémantiques indigènes, les fameuses classifications populaires, pour étudier la connaissance qu'a une société de son environnement ${ }^{4}$. En demandant à l'observateur d'abandonner ses propres catégories, il renouvela ce que l'on allait désormais appeler « les ethnosciences ». La description de systèmes de connaissance parallèles à ceux de la science occidentale a ainsi contribué à reconnaître aux populations non occidentales un savoir qui leur était propre. On peut attribuer à l'anthropologie la paternité d'une pensée sociale des interactions entre sociétés et nature, même écartelée entre un «monisme naturaliste » et un «culturalisme radical» dans son effort d'interprétation ${ }^{5}$. Doit-on pour autant reprocher à ses représentants d'avoir abusé de leur position en associant trop étroitement les cosmogonies natives à des finalités pratiques? La question du caractère conservatoire et durable des pratiques de gestion des populations amérindiennes est largement débattue aujourd'hui, même si l'empathie développée par certains chercheurs vis-à-vis des populations traditionnelles est un fait incontestable. La grande révolution est plutôt liée au fait que les peuples autochtones comme les sociétés locales de la fin du $X X^{\mathrm{e}}$ siècle ont acquis une certaine visibilité en rejoignant l'espace public. Les peuples forestiers ont été «redécouverts " sous le filtre de nouvelles représentations issues de la pensée globale. Ils sont entrés corrélativement dans un processus de bouleversement

\footnotetext{
3 Steward, J.H., 1955. Theory of Culture Change: The Methodology of Multilinear Evolution, Urbana, University of Illinois Press.

4 Conklin, H.C., 1955. Hanunoo color categories, Southwestern Journal of Anthropology, 11, 339-344.

5 Descola, P., 2001. Leçon inaugurale, faite le 29 mars 2001, Collège de France, Chaire d'anthropologie de la nature, [Paris], Collège de France.
} 
radical de leur forme d'organisation politique et sociale en tant qu'acteurs potentiels du développement durable. Les exemples contemporains pris par F. Kohler dans sa partie méthodologique concernent bien ce moment historique de reconstruction du local, mettant en scène dans le cas amazonien des Amérindiens acculturés et des populations traditionnelles postcoloniales. Faisant écho au "retour du paysan » en France, le Brésil a traduit son adhésion au développement durable selon des reconfigurations qui lui sont spécifiques ${ }^{6}$. L'importance de son héritage historique - le traitement politique de la question agraire, d'une part, et celui du multiculturalisme associé au droit des minorités, d'autre part - se manifeste par la constitution de nouvelles figures parées des vertus de notre modernité écologique. Plus généralement, on peut interpréter l'entrée en politique de ces nouvelles catégories d'acteurs, qu'ils soient agriculteurs, peuples de la forêt, populations traditionnelles ou encore occupants d'un terroir, comme une tentative de se réapproprier la gestion du vivant plutôt que de l'abandonner à une ingénierie écologique dont l'efficacité serait aussi à discuter. Il s'agit finalement de parier sur la diversité culturelle plutôt que sur l'homme unidimensionnel, ce que F. Kohler n'aborde pas dans sa première partie, sinon sous couvert d'idéologie.

Je voudrais m'attarder sur les deux aspects développés par F. Kohler, relatifs à la vision culturalisée de la nature, qui soulèvent de façon récurrente le problème de la définition de la biodiversité et du flou de ses contours. «Le premier - écrit-il - dérive de l'inclusion d'espèces animales et végétales domestiquées dans la biodiversité » et au fait que celles-ci ne correspondent pas à la définition symbiotique qu'il en a donnée. Si F. Kohler ne réduit pas la biodiversité, comme c'est souvent le cas, à un inventaire d'espèces, il rejoint par contre l'approche défendue par les conservationnistes anglo-saxons, qui confondent la biodiversité avec la wilderness (nature sauvage) et à ce titre militent pour l'exclusion de toute présence humaine dans les espaces protégés. C'est une position qui est loin d'être partagée par tous les naturalistes et nous convie d'emblée à de nombreuses controverses où se jouent d'abord des rapports de domination culturelle. Si nous raisonnons de façon strictement scientifique, nous devons reconnaître que les catégories de «sauvage » et de « cultivé » sont utiles pour organiser nos connaissances, mais inefficaces dès lors que nous les confrontons à la réalité de ce que nous appelons la biodiversité. Certains de mes amis écologues travaillant sur les plantes utiles amazoniennes ont toujours défendu l'idée d'un gradient reliant le sauvage au domestique, les différences de traitement appliquées aux plantes ne permettant pas de les

\footnotetext{
${ }^{6}$ Cf. Pinton, F., 2009. De la paysannerie française aux peuples contemporains de la forêt amazonienne (Brésil), in Alphandéry, P., Billaud, J.-P. (Eds), La Sociologie rurale en questions, Études rurales, 183, 201-218.
}

ranger dans des cases étanches. Dans un autre ordre d'idée, il peut paraître excessif d'exclure les plantes cultivées de la biodiversité alors même que la question des ressources phytogénétiques est un des grands enjeux de la Convention sur la diversité biologique (CDB). Enfin, les sciences sociales, de leur côté, reconnaissent depuis longtemps la biodiversité comme un objet aux frontières mal définies et contingent des dimensions sociales, économiques et politiques.

Le deuxième aspect concerne la question, très pertinente en soi, du référentiel à considérer pour évaluer un niveau de biodiversité. F. Kohler prend comme exemple le principe de la culture sur brûlis en milieu forestier tropical, étudié par de très nombreux chercheurs qui se concentrent, écrit-il, sur l'existant pour conclure à un enrichissement d'espèces et ne prennent pas en considération un état antérieur qui n'est que supposé. Tous les travaux produits sur le sujet s'accordent pourtant sur le fait que l'agriculture sur brûlis, basée sur l'intégration d'une longue jachère forestière dans le cycle cultural, est parfaitement compatible avec le maintien d'un milieu matriciel forestier. Si les questions d'échelles de temps et d'espace se posent effectivement pour évaluer l'état de la biodiversité et son niveau relatif, on ne peut raisonner aux mêmes échelles pour traiter des problèmes d'extinction des espèces depuis le Néolithique - l'exemple de la faune corse étant mis en avant - et de la corrélation entre biodiversité et diversité culturelle. Les objets ne sont pas les mêmes.

La partie méthodologique développée ensuite par F. Kohler est très instructive. Mais elle invalide paradoxalement le cadre argumentaire défendu jusqu'ici, puisqu'elle se donne pour objet la question de l'évaluation des discours locaux dans un contexte d'élaboration de projets de développement durable. Prenant acte de la dimension politique de la réhabilitation des savoirs traditionnels et du poids des mouvements socioenvironnementalistes au Brésil dans l'évolution des modèles de conservation, F. Kohler propose de « reconsidérer ce que sont les éléments pertinents d'une enquête » à même de questionner la reconnaissance d'une efficacité pratique des cosmologies natives et les «dispositions » d'une population à l'égard de son environnement. Pour ce faire, il ne mobilise guère la figure de la «communauté », figure autour de laquelle l'anthropologie sociale s'est formée, mais plutôt celle de la «microsociété ». La relation local-global n'était pas ou peu explorée par l'anthropologie classique, confortant ainsi le découpage de l'objet observé et gommant l'ouverture des territoires à la multiplicité des flux. Les populations traditionnelles observées par F. Kohler sont ouvertes sur l'extérieur et informées des enjeux nationaux et internationaux de la conservation. Cette disposition implique une analyse de leurs pratiques qui tienne compte de leur articulation à la «modernité ». Elles sont (ou seront) en effet confrontées à des 
dynamiques de «relocalisation » de leurs pratiques et de leurs représentations, sources d'acculturation générant de nouvelles solidarités et de nouveaux conflits. Leur cosmogonie ne peut guère garantir, dans ce contexte, la survie des écosystèmes, comme l'illustre parfaitement l'analyse menée par F. Kohler sur les Indiens Galibi du Nord de l'Amapa. Pour y remédier, il propose de mettre en place une "heuristique de la réalité » qui permette à l'anthropologue engagé dans l'évaluation de projets de conservation d'appréhender la complexité du «système de relations entre humains et monde naturel » et de produire des données exploitables par d'autres disciplines. On ne peut que souscrire à une telle approche, avec la réserve que les objets du savoir et les façons de mesurer ne sont pas non plus à l'abri de préconçus idéologiques.
On ne peut être que réactif au texte de F. Kohler, et c'est une de ses qualités. Il nous incite à revenir sur l'histoire des peuples de la forêt, qui montre sans équivoque que le statut de ces derniers varie au gré des représentations que s'en fait l'Occident et des alliés politiques que ces peuples se font. Elle montre aussi que, si les rapports de pouvoir et de domination formatent la production de connaissance, celle-ci, au gré des époques, revient sans cesse sur ses propres catégories pour reconstruire de nouvelles trames susceptibles de déstabiliser ce que l'on croyait acquis. La reconnaissance de la diversité culturelle nous engage vers la production de nouvelles ontologies qui réintègrent les non-humains dans la sphère des humains. Elle pose la question essentielle de la place que prendront demain les savoirs locaux dans la production de connaissance sur la nature, l'environnement et le vivant. 